\title{
Chemical Model Reduction under Uncertainty
}

\author{
Riccardo Malpica Galassi ${ }^{\mathrm{a} *}$, Mauro Valorani ${ }^{\mathrm{a} *}$, Habib N. Najm ${ }^{\mathrm{b} *}$, \\ Cosmin Safta $^{\mathrm{b} *}$, Mohammad Khalil ${ }^{\mathrm{b} *}$, Pietro Paolo Ciottoli ${ }^{\mathrm{a} *}$

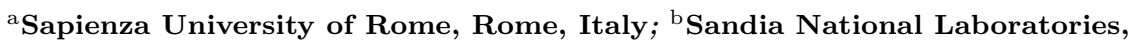 \\ Livermore, CA 94551, USA
}

\begin{abstract}
A general strategy for analysis and reduction of uncertain chemical kinetic models is presented, and its utility is illustrated in the context of ignition of hydrocarbon fuel-air mixtures. The strategy is based on a deterministic analysis and reduction method which employs computational singular perturbation analysis to generate simplified kinetic mechanisms, starting from a detailed reference mechanism. We model uncertain quantities in the reference mechanism, namely the Arrhenius rate parameters, as random variables with prescribed uncertainty factors. We propagate this uncertainty to obtain the probability of inclusion of each reaction in the simplified mechanism. We propose probabilistic error measures to compare predictions from the uncertain reference and simplified models, based on the comparison of the uncertain dynamics of the state variables, where the mixture entropy is chosen as progress variable. We employ the construction for the simplification of an uncertain mechanism in an n-butane-air mixture homogeneous ignition case, where a 176-species, 1111-reactions detailed kinetic model for the oxidation of n-butane is used with uncertainty factors assigned to each Arrhenius rate pre-exponential coefficient. This illustration is employed to highlight the utility of the construction, and the performance of a family of simplified models produced depending on chosen thresholds on importance and marginal probabilities of the reactions.
\end{abstract}

\footnotetext{
${ }^{*}$ Corresponding author

Email address: riccardo.malpicagalassi@uniroma1.it (Riccardo Malpica Galassi ${ }^{\mathrm{a}}$ )
}

Preprint submitted to Combustion and Flame

February 15, 2017

C) 2017. This manuscript version is made available under the Elsevier user license http://www.elsevier.com/open-access/userlicense/1.0/ 
Keywords: Uncertainty Quantification, Reaction mechanisms, Chemical Kinetics Reduction, Autoignition 


\section{Introduction}

Chemical model reduction strategies generally start from a detailed chemical kinetic mechanism as the reference or baseline gold-standard. Given this standard, a specified range of operating conditions or set of state vectors, a select set of Quantities of Interest (QoIs), and a requisite error threshold, a model reduction strategy produces a simplified mechanism of associated size/complexity [1, 2].

This strategy, despite its effectiveness, nonetheless relies on the quality of the starting mechanism. Yet, there is typically significant uncertainty in both the structure of available detailed mechanisms for hydrocarbon fuels, and in their thermodynamic and chemical kinetic rate parameters. Therefore, in principle, the analysis/reduction processes that provide simplified mechanisms starting from the detailed mechanism, and the measures of quality of a simplified mechanism relative to the detailed mechanism, need to account for both model and parametric uncertainties in both mechanisms. This is a challenging, yet highly relevant topic. Overconfidence in the detailed mechanism can lead to a misplaced focus on tight error tolerances in the simplified model, relative to a faulty/uncertain baseline. Simplified model errors ought to be handled along with detailed model uncertainties in the same error budget. Any error norm between simplified and detailed models ought to be weighted appropriately with attendant uncertainties. Moreover, the fact that both the detailed and simplified mechanisms are burdened with uncertainty suggests that any measures of distance between their predictions be done in a probabilistic context. This line of reasoning highlights the need for rethinking model analysis/reduction strategies for uncertain chemical kinetic models.

The above is a significant undertaking with a range of technical challenges. There has been some work addressing model reduction under uncertainty in the context of proper orthogonal decomposition (POD) [3], albeit for small degrees of uncertainty. The dynamical analysis of uncertain ordinary differential equation (ODE) systems has also received some attention $[4,5]$, in a full prob- 
abilistic setting. Further, from the process control perspective, there has also been work $[6,7]$ addressing dynamical systems reduction under parametric uncertainty, relying on balanced truncation $[8,9]$, singular value decomposition, and sensitivity analysis. We note that this work $[6,7]$ presumes parameter variations in intervals, with no probabilistic information. It is not clear, however, how well these methods, traditionally employed for process control in linear or mildly-nonlinear contexts, and, more specifically applied by $[6,7]$ for chemical process and isothermal biochemical systems modeling, extend to the strongly nonlinear thermally activated stiff kinetics of hydrocarbon fuels. It is fair to say that the challenge of dynamical analysis and uncertain chemical model simplification in hydrocarbon kinetics of relevance to combustion has yet to receive significant attention.

We lay out in the following a general strategy for analysis and reduction of uncertain chemical kinetic models, and describe its utilization in the context of ignition of hydrocarbon fuel-air mixtures. The construction is fully probabilistic, allowing for an arbitrary uncertainty structure. It is based on an existing analysis and reduction strategy, using computational singular perturbation (CSP) analysis $[10,11]$, that has been used extensively for deterministic models of hydrocarbon fuels $[1,2]$. We begin in the following with a brief outline of the deterministic simplification strategy, before proceeding to the description of the proposed approach.

\section{Deterministic Simplification Algorithm}

In the CSP-based skeletal reduction algorithm, the criterion for selecting the subset of reactions and species to be retained is based on their relevance to the fast or slow dynamics of a prescribed set of "target" species, the concentration of which is desired to be accurately reproduced by the skeletal mechanism. This algorithm relies on the decomposition of the chemical kinetic processes into fast and slow components, using CSP, and on the identification of the processes that produce the most significant contributions in either the fast or slow 
components $[12,11,13,14]$. As a measure of the degree to which a species contributes to the fast/slow dynamics of the target species, the algorithm adopts the fast/slow CSP Importance Indices, which measure the contribution of each elementary reaction in the detailed mechanism to the fast and slow components of each species production rate $[15,16,17]$. Defining as $(I)_{k_{\text {slow/fast }}}^{i}$ the Importance Index of the $k$-th reaction to the $i$-th species in either the fast or slow subspace and given a user-specified tolerance $\tau$, the $k$-th reaction is included in the simplified mechanism if $(I)_{k_{\text {slow / fast }}}^{i}>\tau$. The rigorous definition of the Importance Indices and the complete algorithm can be found in $[1,2]$. This approach is very useful in generating a spectrum of simplified mechanisms of different sizes, each associated with a given degree of fidelity in predicting chosen QoIs specified by a tolerance on the Importance Indices. More details on the simplification algorithm can be found in $[2,1,18,19]$.

\section{Simplification Strategy under Uncertainty}

Consider a detailed chemical mechanism $\mathcal{M}^{*}(\lambda)$, defined by a set of species $\mathcal{S}^{*}=\left\{S_{1}, \ldots, S_{N}\right\}$ and elementary reactions $\mathcal{R}^{*}=\left\{R_{1}, \ldots, R_{M}\right\}$, where $\lambda$ is the relevant vector of uncertain parameters, e.g. the Arrhenius rate parameters of all reactions. Consider the auto-ignition process of a hydrocarbon fuel-air system in a constant pressure batch-reactor, for a range of initial temperature and stoichiometry, which is used to compute a set of ignition trajectories, providing a database of states $D=\left\{X^{(1)}, \cdots, X^{(K)}\right\}$, where $X \in \mathbb{R}^{N+1}$ is the state vector composed of temperature and the $N$ mole fractions. Given that $\lambda$ is uncertain, let $D_{\lambda}$ denote the database computed for a given value of $\lambda$.

For any given $D_{\lambda}$, and considering a given set of QoIs - such as the set of target species - and a tolerance $\tau$ on Importance Indices [1, 2], the CSP-based analysis and simplification strategy provides a simplified mechanism $\mathcal{M}_{\tau}(\lambda)$, being a subset of the starting mechanism with species $\mathcal{S}_{\tau}(\lambda)$ and reactions $\mathcal{R}_{\tau}(\lambda)$. In fact, given the starting model specification, the simplified model can be specified compactly in terms of a vector of $M$ binary indicators $\alpha^{\tau}(\lambda)=$ 


$$
\begin{aligned}
& \left(\alpha_{1}^{\tau}(\lambda), \ldots, \alpha_{M}^{\tau}(\lambda)\right)^{T}, \text { where } \\
& \qquad \alpha_{r}^{\tau}(\lambda)=\left\{\begin{array}{ll}
1 & \text { for reaction } R_{r} \in \mathcal{R}_{\tau}(\lambda) \\
0 & \text { otherwise }
\end{array}, \quad r=1, \ldots, M\right.
\end{aligned}
$$

In fact, $\alpha^{\tau}(\lambda)$ is a multi-index that specifies $2^{M}$ models. We can view the process of database generation, analysis, and model simplification as an input-output map:

$$
f_{\tau}(\lambda): \lambda \rightarrow \alpha^{\tau}(\lambda)
$$

which provides a convenient abstraction for the use of uncertainty quantification (UQ) methods to account for uncertainty in $\lambda$ in the process of simplified model selection.

Placing ourselves in a probabilistic UQ setting, uncertain quantities are represented as random variables. Accordingly, $\lambda$ is defined as a real-valued random vector with a presumed joint Probability Density Function (PDF) $p(\lambda)$. The specification of this PDF is a major challenge in general, requiring recourse to available data on each parameter in the model, and allowing proper accounting for the correlation among different uncertain parameters. We discuss this challenge, as regards chemical mechanisms for combustion, later below in $\S 5$.

Generating $n$ random samples from $p(\lambda),\left\{\lambda^{(1)}, \cdots, \lambda^{(n)}\right\}$, the input-output map of Eq.(2) provides corresponding samples $\left\{\alpha^{\tau j}\right\}_{j=1}^{n}$, where $\alpha^{\tau j}=\alpha^{\tau}\left(\lambda^{(j)}\right)$, so that we can estimate, $\forall \alpha=\left(\alpha_{1}, \cdots, \alpha_{M}\right)$, the joint probabilities,

$$
P_{\tau}(\alpha) \approx \frac{1}{n} \sum_{j=1}^{n} \delta_{\alpha^{\tau j} \alpha}
$$

where $\delta_{\alpha^{\tau j} \alpha}$ is the Kronecker delta,

$$
\delta_{\alpha^{\tau j} \alpha}= \begin{cases}1 & \text { if } \alpha=\alpha^{\tau j} \\ 0 & \text { otherwise }\end{cases}
$$

Thus, the contribution of each sample $j$ to the sum for $P_{\tau}(\alpha)$ in Eq. (3) is 1 if $\alpha^{\tau j}=\alpha$, and 0 otherwise. Further, we have

$$
\delta_{\alpha^{\tau j} \alpha}=\prod_{i=1}^{M} \delta_{\alpha_{i}^{\tau j} \alpha_{i}} .
$$


The joint probabilities provide a wealth of information on the coupling among reactions. For example, marginalizing over $M-2$ reactions, provides the 2-way joint probabilities for any two given reactions $(p, q)$,

$$
P_{\tau}\left(\alpha_{p}, \alpha_{q}\right) \approx \frac{1}{n} \sum_{j=1}^{n} \delta_{\alpha_{p}^{\tau j} \alpha_{p}} \delta_{\alpha_{q}^{\tau j} \alpha_{q}}
$$

This provides information on the relevance of two reactions $p$ and $q$ being included/excluded jointly or separately in the model. Similarly, this analysis can be generalized to any subset of reactions forming a pathway of interest. Moreover, extending the scope to a full sub-mechanism, the joint picture provides a statement concerning the probability of any given mechanism that is a subset of the detailed model. Given a reasonable search strategy on $\alpha$, one can thus select the model with the highest $P(\alpha)$ as the one most supported by the reduction strategy. Alternatively, if multiple models have comparable probability, a Bayesian model averaging $[20,21]$ strategy can be employed to provide a pooled/average prediction.

Given the complexity of the joint-picture, and the need for large numbers of samples to establish multivariate statistics, we confine ourselves here to the marginal probabilities for individual reactions,

$$
P_{\tau}\left(\alpha_{i}\right) \approx \frac{1}{n} \sum_{j=1}^{n} \delta_{\alpha_{i}^{\tau j} \alpha_{i}}, \quad i=1, \ldots, M .
$$

With this, and since $\delta_{\alpha_{i}^{\tau j} 1} \equiv \alpha_{i}^{\tau j}$, the marginal probability that a reaction is included in the simplified mechanism for a given $\tau$, is given by

$$
\mathcal{P}_{i}^{\tau}=P_{\tau}\left(\alpha_{i}=1\right) \approx \frac{1}{n} \sum_{j=1}^{n} \alpha_{i}^{\tau j} .
$$

In this way, we arrive at a proposed strategy for model reduction under uncertainty, whereby a reaction is included in the simplified mechanism for a given $\tau$, when its marginal probability satisfies $\mathcal{P}_{i}^{\tau}>\theta$, where $0<\theta<1$ is a userspecified threshold.

We note, of course, that this marginalization is applied to samples, each of which is a mechanism selected based on CSP analysis and the associated 
simplification strategy for the chosen sample of the Arrehnius parameter vector of the detailed mechanism. Thus, if, say, partial equilibration or other dynamical arguments strongly require that a given pair/tuple of reactions be included together or not at all, then when one of these reactions is included with high/low marginal probability, the other(s) will be as well. As a result, these pairings/groupings will be implicitly respected in the marginally selected mechanism. Accordingly, the predominant impact of the limitation of the present scope to marginal statistics is that we forego, for the present, explicit discovery/discussion of such groupings where they do exist.

\section{Error measures}

An a-posteriori error analysis is needed to assess the quality of a given simplified mechanism because the highly non linear character of the system of interest renders it impossible to derive a-priori error estimates. In general, we look for simplified mechanisms that are both computationally efficient, i.e. made up of a small number of species, and accurate in replicating some relevant features of the predictions from the detailed mechanism, which features we term as QoIs, such as the ignition delay time and the transient evolution of the species of interest. The presence of uncertainty in the reaction rates of the detailed mechanism adds complexity to the choice: the evaluation of the simplified mechanism's features has to be pursued in a probabilistic framework.

The discrepancies between ignition trajectories $X_{i}^{d}(t)$ and $X_{i}(t), i=1, \ldots, N+$ 1 , generated by detailed and simplified models respectively, can be assessed with different metrics. The two most natural choices involve errors among solution trajectories (i) in the $\left\{t, X_{i}(t)\right\}$ phase space, and/or (ii) in the $\left\{\psi(t), X_{i}(t)\right\}$ phase space, where $\psi$ is a suitable progress variable and time $t$ acts as a parametric abscissa.

The $\left\{t, X_{i}(t)\right\}$ phase space is suitable for ignition delay time measurements. A deterministic relative error on ignition delay time may be defined as:

$$
E_{\mathrm{ign}}=\frac{t_{\mathrm{ign}}-t_{\mathrm{ign}}^{d}}{t_{\mathrm{ign}}^{d}}=\frac{\Delta t_{\mathrm{ign}}}{t_{\mathrm{ign}}^{d}}
$$


where $t_{\mathrm{ign}}^{d}$ is the ignition delay of the detailed mechanism-based prediction. However, in our probabilistic framework, we have uncertain predictions $X_{i}(t, \lambda)$ for each component of the state vector, based on any simplified model, and $X_{i}^{d}(t, \lambda)$ based on the detailed model. Error measures may thus be obtained by comparing the ignition delay times on a sample-by-sample basis, obtaining samples of the relative errors and, in turn, its probabilistic distribution, for any given simplified model. Then, relevant statistics can be compared, such as averages, standard deviations, or quantiles.

On the other hand, Valorani et. al. [22] observed that, because of the exponential dependence of the ignition delay time on model parameters, the error in solution trajectories measured in the $\left\{t, X_{i}(t)\right\}$ plane can exhibit non-monotonic convergent behavior with decreasing degree of simplification of the kinetic mechanism. This non-monotonicity comes about as a consequence of the elimination in total or in part of chain branching/propagation reactions that can promote or postpone the onset of ignition. It was concluded in [22] that an error measure involving an integral measure of the distance between ignition trajectories in the $\left\{\psi, X_{i}\right\}$ phase space exhibits the sought-after monotonic convergent behavior with decreasing degree of simplification.

An appropriate progress variable $\psi(t)$ should provide a monotone and non singular mapping of time $t$ versus $\psi$, so that all mappings involving the components $X_{i}$ of the state $X$ are one-to-one functions of $\psi$.

We introduce a progress variable based on the mixture entropy change. The entropy per unit mass, $s$, of a mixture of $N$ ideal gases is given by

$$
\begin{aligned}
& s\left(T, p, Y_{j}\right)=\frac{S\left(T, p, X_{j}\right)}{\bar{W}} \\
& S\left(T, p, X_{j}\right)=\sum_{j=1}^{N}\left(S_{j}^{0}(T)-\mathcal{R} \log \left(\frac{p}{p_{\text {ref }}}\right)-\mathcal{R} \log X_{j}\right) X_{j}, \\
& S_{j}^{0}(T)=\Delta S_{f, j}^{0}+\int_{T_{\text {ref }}}^{T} \frac{\bar{C}_{p, j}(T)}{T} d T,
\end{aligned}
$$

where $S$ is the entropy per molar units, $\Delta S_{f, j}^{0}$ the entropy species formation per molar units, $\bar{C}_{p, j}$ the species molar heat capacity, $T_{\text {ref }}$ and $p_{\text {ref }}$ are the 
reference temperature and pressure at standard conditions, and $\mathcal{R}$ the universal gas constant. This mixture entropy can change because of both external and internal irreversible processes, that is:

$$
d S=d S_{\text {ext }}+d S_{i n t},
$$

where $d S_{\text {ext }}$ accounts for heat exchange with the surroundings and the entropy change due to internal processes $d S_{\text {int }}$ is evaluated according to the following differential relation:

$$
d S_{\text {int }}=-\frac{1}{T} \sum_{j=1}^{N_{s}} \mu_{j} d X_{j},
$$

where $\mu_{j}$ is the chemical potential per molar units of the $j$-th species defined as

$$
\mu_{j}\left(T, p_{j}\right):=H_{j}(T)-T S_{j}\left(T, p_{j}\right),
$$

$p_{j}$ is the partial pressure, $N_{j}$ is the number of moles of the $j$-th species and $H_{j}$ is the species enthalpy per molar units given by

$$
H_{j}(T)=h_{j}(T) W_{j}=\left(h_{j}^{0}(T)+\int_{T_{\mathrm{ref}}}^{T} c_{p, j}(T) d T\right) W_{j} .
$$

In isolated systems, where there is no interaction at all with the surroundings, $d S_{\text {ext }}=0$ and the overall entropy production is due to $d S_{i n t}$, whereas the presence of heat exchange, even if the system is closed (no mass exchange), makes the term $d S_{\text {ext }}$ different from zero. Furthermore, it can be proved that $s_{\text {int }}$ is monotone and attains a maximum at equilibrium, making it a suitable progress variable. Thus, we define:

$$
\hat{s}(t):=\int_{0}^{t} d s_{i n t},
$$

where $s$ is the mixture entropy per unit mass and $d s_{\text {int }}$ is the mixture entropy change due to internal processes.

Next, we scale $\hat{s}(t)$ to get:

$$
\tilde{s}(t):=\frac{\hat{s}(t)-\hat{s}(0)}{\hat{s}\left(t_{\text {final }}\right)-\hat{s}(0)} .
$$


In a deterministic context, trajectory errors in the $\left\{\psi, X_{i}\right\}$ space are usually obtained as integral measures of the distance between the predictions from the detailed and simplified models.

Again, in our probabilistic framework, we have uncertain predictions $X_{i}(\tilde{s}, \lambda)$ for each component of the state vector, based on any simplified model, and $X_{i}^{d}(\tilde{s}, \lambda)$ based on the detailed model. Probabilistic distributions of the distances can be obtained by comparing the uncertain predictions on a sample-by-sample basis, giving way to the production of a number of probabilistic results such as averages, standard deviations, or quantiles of the distance-based error.

Other compact probabilistic error measures in the $\left\{\psi, X_{i}\right\}$ plane may be obtained comparing directly the averages and standard deviations of the uncertain trajectories produced with the detailed mechanism and any given simplified mechanism, as follows. First we introduce the mean $(\mu)$ and standard deviation $(\sigma)$ functions for the set of trajectories generated by the $\lambda$ samples as:

$$
\begin{array}{ccc}
\mu_{i}(\tilde{s})=\mathrm{E}\left[X_{i}(\tilde{s}, \lambda)\right], & \mu_{i}^{d}(\tilde{s})=\mathrm{E}\left[X_{i}^{d}(\tilde{s}, \lambda)\right], & i=1, \ldots, N+1 \\
\sigma_{i}(\tilde{s})=\left(\mathrm{V}\left[X_{i}(\tilde{s}, \lambda)\right]\right)^{\frac{1}{2}}, & \sigma_{i}^{d}(\tilde{s})=\left(\mathrm{V}\left[X_{i}^{d}(\tilde{s}, \lambda)\right]\right)^{\frac{1}{2}}, & i=1, \ldots, N+1
\end{array}
$$

where $\mathrm{E}[\cdot]$ is the expectation, and $\mathrm{V}[\cdot]$ is the variance, both over $\lambda$. We then define the weighted relative error norms, with $w_{i}(\tilde{s}) \equiv 1 / \sigma_{i}^{d}(\tilde{s})$

$$
E_{\mu_{i}}^{p} \equiv \chi_{p, w_{i}}\left(\mu_{i}, \mu_{i}^{d}\right) \approx \frac{\left(\sum_{k=1}^{K} w_{i}\left(\tilde{s}_{k}\right)\left|\mu_{i}\left(\tilde{s}_{k}\right)-\mu_{i}^{d}\left(\tilde{s}_{k}\right)\right|^{p}\right)^{\frac{1}{p}}}{\left(\sum_{k=1}^{K} w_{i}\left(\tilde{s}_{k}\right)\left|\mu_{i}^{d}\left(\tilde{s}_{k}\right)\right|^{p}\right)^{\frac{1}{p}}} .
$$

where $K$ is the number of discrete points. This measure quantifies the distance between the average transient evolution of the $i$-th species concentration computed with a detailed and a reduced model, giving a larger weight to the distance where the standard deviation of the detailed model is smaller. Similarly, we define:

$$
E_{\sigma_{i}}^{p} \equiv \chi_{p, w_{i}}\left(\sigma_{i}, \sigma_{i}^{d}\right)
$$

where the comparison is between the standard deviations. Errors for each species/temperature can be averaged over all species/temperature, and/or over 
the target species, the latter being a useful measure to rank the quality of the simplified mechanisms, given that, by construction, the skeletal mechanisms are tailored for the target species. Thus, the compact error measures on averages and standard deviations are:

$$
E_{\mu_{\mathrm{tgt}}}^{p}=\frac{\sum_{i=1}^{N_{\mathrm{tgt}}} E_{\mu_{i}}^{p}}{N_{\mathrm{tgt}}},
$$

and

$$
E_{\sigma_{\mathrm{tgt}}}^{p}=\frac{\sum_{i=1}^{N_{\mathrm{tgt}}} E_{\sigma_{i}}^{p}}{N_{\mathrm{tgt}}}
$$

where the sum is extended to the target species only.

\section{Application to an n-butane mechanism}

We next describe the utilization of the above construction for simplification of a 176-species, 1111-reaction, uncertain n-butane-air mechanism with published uncertainty for Arrhenius rate parameters [23], based on constant pressure homogeneous ignition. We explore convergence of the results as a function of the number of random samples, and examine errors in ignition delay time and uncertain trajectories with respect to the detailed mechanism.

The specification of uncertain parameters in [23] is in the form of temperature dependent uncertainty factors $F_{r}(T)$ for the Arrehnius forward rate $k_{r}(T)$ of each reaction $r=1, \ldots, M$. Uncertainty factors have been employed traditionally in the combustion literature [24] to specify the uncertainties on individual reaction pre-exponential constants $A_{r}$, where $F_{r} \equiv A_{r, \max } / A_{r, \text { nom }} \equiv$ $A_{r, \text { nom }} / A_{r, \min }$, and the max and min values are some stated quantiles on a presumed log-normal distribution ${ }^{1}$ for each reaction [25]. The temperature dependent $F_{r}(T)$ in [23] is defined similarly in terms of $k(T)$, employing $2 \sigma$ quantiles. Given the paucity of data, a single functional form $F(T)=F_{300 \mathrm{~K}} \exp \mid g$. $\left(T^{-1}-300^{-1}\right) \mid$ is presumed [23], based on [26], specified with provided values for $F_{300 \mathrm{~K}}$ and $g$ for each reaction, where $T$ is temperature in $\mathrm{K}$. In principle,

\footnotetext{
${ }^{1} \mathrm{~A}$ lognormal is presumed to ensure Arrhenius rate positivity by construction.
} 
allowing for temperature-dependent uncertainty factors on $k(T)$ corresponds to presuming some uncertainty in the temperature exponent and/or the activation energy, aside from that in the pre-exponential. One can, indeed, use such a given $F(T)$ to constrain a presumed multivariate Gaussian on $(\ln A, E, n)$ for each reaction [27]. However, it may be argued that the correlation structure among the Arrhenius rate parameters should, in principle, be informed by available experimental data on each reaction and other experimental details, rather than a presumed functional form for $F(T)$. This concern, along with the desire to avoid tripling the dimensionality of the uncertain input space, lead us to ignore the temperature dependence of the uncertainty factors in the present study. We certainly do affirm the importance of accounting for parametric correlations in chemical models, nonetheless we feel they may be safely ignored in the present context, as further discussed in the following paragraph. Thus, we pick a representative temperature of $1500 \mathrm{~K}$, and set the uncertainty factor as a constant $F \equiv F(1500 \mathrm{~K})$ throughout the ignition process, based on the given $F(T)$ from [23], for each reaction. Accordingly, we account for uncertainty only in the pre-exponential rate constants $A$, and ignore uncertainty in $(E, n)$. Thus, to be precise, and in view of the earlier formulation above, we define the uncertain-parameter vector $\lambda$ as composed of the Arrhenius pre-exponential rate constants, $\lambda=\left(A_{1}, \cdots, A_{M}\right)$, where each $A_{r}$ is presumed independent and lognormally distributed. The lognormal distributions are specified based on the given nominal $A$ value and $F(1500 \mathrm{~K})$ for each reaction.

Clearly, this construction, aside from ignoring the correlation among uncertain parameters $(A, n, E)$ of each reaction, also ignores any correlation between rate parameters of different reactions. This is also the case in [23], and, in fact, in most mechanism-scale uncertainty analyses in the combustion literature. While examples of inferred joint densities on rates of multiple reactions [28, 29], mechanism-scale constrained parametric domains [30, 31, 32], and correlations among uncertain rates of numerous reactions [33], do exist, it is fair to say that published information on chemical kinetic mechanisms of combustion relevance never includes the specification of the joint PDF on all uncertain parameters in 
the model. Most typically, published mechanisms include only a specification of constant uncertainty factors for each reaction. Yet, correlations arise naturally from data fitting, and their importance in kinetic models is well acknowledged $[34,35,36,37]$. When a rate constant is estimated from an experiment, where the fitting model relies on other uncertain reaction rate parameters, or, say, on uncertain thermodynamic properties, the fitting naturally induces a correlation among all these parameters. We stress, nonetheless, that our choice in the present work, where we only account for uncertainties in the pre-exponentials and ignore uncertainties in $(n, E)$ as well as correlations among rate parameters of different reactions, does not impact the generality of the strategy outlined in this study. Correlations can be easily included in future work. The present strategy for chemical model reduction under uncertainty can, indeed, employ any joint parametric PDFs where available.

\subsection{Problem setup}

We set up the homogeneous, constant pressure, n-butane-air mixture adiabatic ignition problem with a deterministic initial condition corresponding to a stoichiometric mixture at $1050 \mathrm{~K}$ and $1 \mathrm{~atm}$. It is clear that a range of initial conditions would allow the exploration of the phase space more exhaustively, and the generation of more comprehensive simplified mechanisms, however, for now, we limit this study to one initial condition in order to emphasize the key aspects of the reduction strategy. For any given sample of $\lambda$, we compute an ignition trajectory by integrating the governing system of stiff ordinary differential equations using CVODE [38], employing TChem [39] for evaluation of chemical source terms and analytic Jacobians. Each integrated trajectory provides a database of states, based on which the CSP-based simplification method generates one simplified mechanism for each specified tolerance $\tau$ on Importance Indices. We define the $\boldsymbol{\tau}$ vector as a uniformly-spaced list of $10 \tau$ tolerances on Importance Indices between 0.02 and 0.27 , which proved to generate a widespread family of simplified mechanisms in a preliminary deterministic analysis. We define the set of target species as $\left(\mathrm{C}_{4} \mathrm{H}_{10}, \mathrm{O}_{2}, \mathrm{CO}_{2}, \mathrm{H}_{2} \mathrm{O}, \mathrm{O}, \mathrm{OH}\right.$, 
$\mathrm{CO})$. Given this setup, we generate $n$ random samples of $\lambda$, then we integrate to obtain $n$ ignition trajectories and produce the corresponding samples of integer vectors $\left\{\alpha^{\tau j}\right\}_{j=1}^{n}, \forall \tau$, to estimate the marginal probabilities that each reaction is included in the simplified mechanisms. Finally, we use the proposed strategy to generate simplified mechanisms for different thresholds $\theta$ on marginal probabilities. We examine the effects of the thresholds $\theta$ on the simplified mechanism and on the a-posteriori errors. We also illustrate the utility of the probabilistic reduction strategy, in particular, when employing the resulting simplified mechanisms in a deterministic prediction context, which is what a general user might be interested in.

\subsection{Uncertain predictions of the detailed mechanism}

Uncertainty in the Arrhenius parameters results in uncertain detailed autoignition trajectories as shown in Fig. 1 for select species/temperature. In these plots, the average trajectory of a species concentration/temperature is plotted against the progress variable defined in section 4. Also plotted are two curves that lie \pm 2 standard deviations away from the mean prediction. The choice of examining uncertain trajectories with respect to the progress variable allows us to avoid artifacts associated with the fast growth of uncertainty due to small shifts of ignition time, during the ignition regime. The trajectories of temperature and $\mathrm{O}_{2}$ (not shown) are the least affected by uncertainty among those included in the target set. Except for the fuel, which is depleted early, the largest predictive uncertainties on species concentrations occur in the proximity of $\tilde{s}=0.9$, which represents the mixture evolution roughly halfway through the ignition.

Figure 2 shows the behavior of temperature in time for a number of samples, highlighting the variability in the ignition delay time. Note that there is no uncertainty in the equilibrium point. This is an expected result, given that equilibrium is determined by the equilibrium constants, which are sole functions of the thermodynamic properties of the mixture, that are not assumed as uncertain in this application. Thus, uncertainty appears only in the transient response. 
Also shown in Fig. 2 is the evolution of the progress variable $\tilde{s}$ in time for a number of samples. Its monotone and non-singular nature even when approaching equilibrium is highlighted in the box in the bottom right corner. Further, we note that, while these results, being at $1 \mathrm{~atm}$, are not directly comparable to the $10 \mathrm{~atm}$ ignition delay results in [23], we have also ran our analysis at $10 \mathrm{~atm}$, arriving at a mean ignition delay time of $5.4 \mathrm{~ms}$ and a standard deviation of $1.1 \mathrm{~ms}$, both of which are consistent with the findings in [23].

\subsection{Monte Carlo convergence}

We now examine the convergence of the Monte Carlo (MC) error to estimate the number of samples required to produce accurate enough $\left\{\alpha^{\tau}\right\}$ vectors. In general, for a given target accuracy, the number of required sample simulations scales with the number of important uncertain parameters, which is typically small, but is not known a-priori. The MC error in the estimation of the marginal probability of reaction $r, \mathcal{P}_{r}^{\tau}$, for any number of samples $n$, defined as $\varepsilon=\mathcal{P}_{r, n}^{\tau}-$ $\mathcal{P}_{r}^{\tau}$ should decay as $n^{-1 / 2}$. The convergence rate of the error can be estimated by looking at the decay of the incremental error defined as $\epsilon_{r, n}^{\tau}=\mathcal{P}_{r, n}^{\tau}-\mathcal{P}_{r, 2 n}^{\tau}$, $r=1, M$, for the geometric series $n=k, 2 k, 4 k, \ldots$, given a starting sample size $k$. For each $n$, we compute the error corresponding to equal-sized subsets of different combinations of $\left\{\alpha^{\tau j}\right\}$ realizations. Figure 3 shows the scatter of the maximum $\mathrm{MC}$ error over the $M$ reactions for increasing numbers of samples. The average MC error decays as $n^{-1 / 2}$ as expected based on the Central Limit Theorem. We will employ $n=10000$ samples in what follows, to keep the maximum error on statistics below $1 \%$.

\subsection{Mechanism Simplification}

We next perform the simplification of the uncertain n-butane-air mechanism with the specified $10 \tau$ tolerances on Importance Indices. Figure 4 shows the number of retained species/reactions with increasing $\theta$, for all the prescribed $\tau$ thresholds. As expected, the increase in $\tau$ acts towards a reduction in the number of species/reactions included in the simplified mechanisms. The role of 
$\theta$ is similar to that of $\tau$ in its consequence, namely the reduction of the retained reactions, although it operates on the probabilistic side of the analysis. In fact it deals with the probabilities of inclusion of each reaction in the reduced mechanism, given the specified parametric uncertainties in the detailed mechanism. For any fixed value of $\tau$, and for any given reaction $r$, each random parameter vector sample $\lambda^{(j)}, j=1, \ldots, n$, leads to a $1 / 0$ choice on the inclusion/exclusion of this reaction, with the resulting marginal probability estimated as the sample average $\mathcal{P}_{r}^{\tau} \approx \mathcal{P}_{r, n}^{\tau}$. The final decision on inclusion/exclusion of reaction $r$ then depends on $\theta$, and specifically on whether $\mathcal{P}_{r}^{\tau}>\theta$. A low value of $\theta$ allows the inclusion of reactions that are marked as important/included in only a minority of the samples, and thus have a relatively low inclusion probability $\mathcal{P}_{r}^{\tau}>\theta$, while for high values of $\theta$ only highly probable reactions are included. Obviously, $\theta=1$ means that a reaction is included only if it is found to be included for every randomly sampled $\lambda^{(j)}$. Thus, this limit of $\theta$ would be most exclusive, resulting in the smallest set of included reactions for the given $\tau$. On the other hand, a $\theta=0$ allows for the inclusion of the majority of reactions, namely, any reaction that is selected for at least one sample of $\lambda^{(j)}$ is included. We also observe that the quantitative effect of $\tau$ in decreasing the number of retained species/reactions in this application is generally much stronger than that of $\theta$. The maximum change in the number of retained species that has been obtained varying $\theta$ through its whole excursion range, that is from 0 to 1 , is approximately 60 and happens for the case of $\tau=0.047$ (depicted in green in Fig 4). However, in all the other $\tau$-cases considered, the effect of $\theta$ is of the order of 20 species or less. Figure 5 shows the histogram of the probability of inclusion $\mathcal{P}_{r}^{\tau}$ for a representative choice of values for $\tau$. As Fig.4 suggests, with the non-smooth behavior of the number of active species/reactions with increasing $\theta$, where sudden jumps are observed together with long plateaus, $\mathcal{P}_{r}^{\tau}$ has the tendency to assume only certain values. There are wide intervals of values of $\mathcal{P}_{r}^{\tau}$ that are never taken by any reaction. For example, in the case of $\tau=0.020$, no reactions have a probability of inclusion which falls between 0.1 and 0.3 , and 0.4 and 0.6 . Moreover, many reactions have a probability of 
inclusion which is equal, or very close to, 1 . These groups of reactions are those whose relative importance is not sensitive to the uncertainty in the Arrhenius parameters and, for the given $\tau$, will be labeled as active/included for any choice of $\theta$. The only one case in which this behavior is less evident is again $\tau=0.047$, where the group of reactions with $\mathcal{P}_{r}^{\tau} \approx 1$ is smaller and the histograms are more uniformly distributed. These results help to give more insight into the system's behavior when it is subject to perturbations in its parameters. For almost all the $\tau$ thresholds employed, there is a bulk of reactions that is always included in spite of the trajectories variability.

\subsection{Error Analysis}

For the a posteriori error analysis, we compare 8 sets of simplified mechanisms, built using 8 probabilistic thresholds $\theta$, namely $(0.1,0.3,0.4,0.5,0.7$, $0.8,0.9,0.99)$, each set including 10 mechanisms with different levels of fidelity according to the $\tau$ tolerances. The probabilistic thresholds have been chosen in order to explore the most interesting $\mathcal{P}_{r}^{\tau}$ ranges based on the results showed in Fig.5.

Each simplified mechanism is identified by the pair $(\tau, \theta)$ that has been used to generate it, as explained in section 3. Several QoIs can be drawn a posteriori to evaluate their degree of accuracy with respect to the detailed mechanism, as described in section 4 .

Consider first the ignition delay time. Previous experience with deterministic chemical model analysis/reduction with CSP [22] demonstrated a general lack of monotonicity of the ignition delay time, and its error with respect to that based on the detailed mechanism, when reducing the number of species/reactions by varying the CSP threshold $\tau$. This is found here as well. Figure 6 shows the predicted ignition delay times with their error bars, compared to the uncertain prediction based on the detailed mechanism. All the 10 sets shown, each comprising 8 mechanisms corresponding to the same $\tau$ threshold, are plotted against the number of retained species. The $\theta$ threshold increases from right to left in each set. A logarithmic scale is used in the x-axis for the sake of clarity, since 
the majority of the resulting mechanisms are in the region of 30 to 60 species. As a general result, the ignition delay time remains acceptable, in both average and standard deviation, until around 60 species, then it becomes irregular, being above or below the detailed prediction, without exhibiting a monotonic tendency towards promoting or postponing the onset of ignition by eliminating species/reactions.

A global monotonic trend is instead observed in the $95 \%$ quantiles of the distributions of the relative error between the detailed and the simplified predictions of the ignition delay time, as shown in Fig.7. The distributions are built by evaluating the relative error of each simplified mechanism-based random ignition with respect to the corresponding detailed mechanism-based ignition. As already pointed out, errors are relatively small for mechanisms involving more than 60 -species. Further, despite islands of local non-monotonicity, we can generally say that increasing $\tau$ and/or $\theta$ leads to higher errors in ignition delay times.

Consider next a posteriori errors measured by trajectory error norms. Figures 8 and 9 show the probabilistic error norms obtained as per Eqs.(21) and (22), that are averaged over the target species only. In particular, we present the un-weighted $L_{2}$ error in averages and standard deviations, plotted against the number of species for each $\tau$-set, parametrized with $\theta$. In both plots, we see reasonably clear global trends towards higher error with increased $(\tau, \theta)$. Nonetheless, local non-monotonicity is also observable in these results. Removing one reaction at a time from a chemical model, even as guided by the dynamical analysis information from CSP, does not monotonically cause increase in error. This is because of the complex network structure underlying the model. In particular, it may be imagined that removing a whole reaction sub-network can potentially provide a more physically-valid reaction mechanism than simply removing part of it.

Beside this, Figs. 8 and 9 indicate that average probabilistic errors for target species, including both means and standard deviations, are acceptable over a wide range of importance index tolerances and probabilistic thresholds. The 
(relative) errors in the means span roughly $0.05 \%$ to $10 \%$, depending on $(\tau, \theta)$, while those in the standard deviations are larger, spanning $0.5 \%$ to $200 \%$. Evidently, less severe simplification can be tolerated for similar accuracy in standard deviations versus the means. Thus, larger mechanisms are necessary for capturing both predictive means and uncertainties to the same degree of accuracy. Again, mechanisms down to 60 species show acceptable error figures in both averages and standard deviation accuracy with respect to the uncertain detailed mechanism.

We also examined the behavior of other norms, including both weighted and unweighted $L_{2}, L_{1}$, and $L_{\infty}$ norms. Among all these, the unweighted $L_{2}$ errors exhibited the clearest global trends in $(\tau, \theta)$, hence their inclusion here. The other plots do not go against the illustrated global trends, they simply exhibit more local non-monotonicity. In particular, we suspect that weighted error norms exhibit higher local non-monotonicity because of the role of the

weight, namely the standard deviation along the orbital predictions based on the detailed mechanism, which adds an extra level of complexity given its additional dependence on the database of solutions and sampling noise.

\section{A motivation question}

We consider next the following question: what is the advantage that a general user, who might not be ncessarily interested in uncertain predictions but rather in deterministic predictions from robust reduced mechanims, can derive from the use of a simplified mechanism generated through the proposed probabilistic approach. A general user would choose the suitable simplified mechanism for his/her applications by trading off accuracy for improvements in computational costs, i.e. a lower number of retained species/reactions. We presume that he/she are further interested in ensuring some degree of robustness in the simplified model, in that its deterministic prediction is within requisite thresholds with sufficiently high probability, given the existing uncertainties in the detailed mechannism. However, again, he/she would employ this simplified mechanism 
in a deterministic context, that is by setting the uncertain parameters to the nominal values. We will illustrate below that the uncertainty-based construction of the simplified mechanisms ensure that, even when the mechanism is employed with nominal parameters, it has a lower probability of violating its presumed error thresholds, in comparison to the alternative option of deterministic reduction ignoring uncertainty.

In a general setting, let $y=f(\lambda)$ be the detailed forward model, where $\lambda$ is a vector of random variables having a joint distribution $p_{\lambda}$, whose nominal values are $\lambda_{0}$. We recognize two possible approaches to obtain a simplified forward model. The first one is the deterministic approach for which the simplified model is a function of the nominal value of the uncertain parameter:

$$
\tilde{y}=g\left(\lambda_{0}\right)
$$

The simplified model $g\left(\lambda_{0}\right)$ is chosen a-posteriori, based on an error requirement of the kind:

$$
E_{1}=\left\|g\left(\lambda_{0}\right)-f\left(\lambda_{0}\right)\right\|<\epsilon
$$

where $\epsilon$ is a user-defined error threshold.

The issue with this approach is that it might lead to underestimating the error with respect to reality, if $\lambda$ is in fact uncertain, and it happens that the true value of $\lambda$ is quite different from $\lambda_{0}$. In fact, if the "true" value of $\lambda$ is $\lambda^{\star}$, and we define:

$$
E_{2}=\left\|g\left(\lambda_{0}\right)-f\left(\lambda^{\star}\right)\right\|
$$

there is the possibility that $E_{2}>\epsilon>E_{1}$, which means that the simplified model $g\left(\lambda_{0}\right)$ can be farther from reality than the user intended by the $\epsilon$-certification, with some probability.

Clearly, we don't know the value $\lambda^{\star}$. However, given the uncertain $\lambda$, modeled as a random variable, we have that $E_{2}(\lambda)=\left\|g\left(\lambda_{0}\right)-f(\lambda)\right\|$ is a random variable, and the deterministically reduced model $g\left(\lambda_{0}\right)$ has a certain probability of missing the threshold of error $(\epsilon)$ that the user tried to enforce. In other words, predictions with $g\left(\lambda_{0}\right)$ produce an actual error $E_{2}(\lambda)$ relative to the detailed model where the probability $P\left[E_{2}(\lambda)>\epsilon\right]>0$. 
In contrast, the approach presented in this paper considers $\lambda$ as a random variable up-front and generates probabilistic simplified models of the kind:

$$
\hat{y}=h(\lambda) \quad \lambda \sim p_{\lambda}
$$

The simplified model is chosen a-posteriori, based on probabilistic error constraints such as those defined in section 4, that treat the models in a probabilistic framework, i.e. by comparing averages and standard deviations, or by building probabilistic distributions of the predictions. However, a general user would presumably employ this model for deterministic predictions. In this case:

$$
\hat{y}_{0}=h\left(\lambda_{0}\right)
$$

and the error between this model and reality is:

$$
E_{3}^{*}=\left\|h\left(\lambda_{0}\right)-f\left(\lambda^{\star}\right)\right\|
$$

Again, given that the true $\lambda^{\star}$ is unknown, we can only employ

$$
E_{3}(\lambda)=\left\|h\left(\lambda_{0}\right)-f(\lambda)\right\|,
$$

which is a random variable. It will be shown in the following that

$$
P\left[E_{2}(\lambda)>\epsilon\right]>P\left[E_{3}(\lambda)>\epsilon\right] .
$$

This means that the mechanisms generated with our probabilistic approach have a lower chance of missing the user-enforced threshold $\epsilon$ when compared against an uncertain detailed mechanism, even if employed with nominal uncertain parameters, with respect to the mechanisms generated ignoring the uncertainty.

Figure 10a shows the PDF of $E_{3}(\lambda)$ for one of the obtained simplified mechanisms, together with its error certification $\epsilon$, already presented in Fig.8. $E_{3}(\lambda)$ is calculated in the $\left\{\psi, X_{i}\right\}$ phase space and is averaged over the target species only. The probability of missing the error certification is as low as $8 \%$. On the contrary, the PDF in Fig.10b is the PDF of $E_{2}(\lambda)$ for a simplified mechanism generated with a classical, non-probabilistic, method, that has a similar error 
certification $\epsilon$, enforced on classical, deterministic, error figures. The probability of missing the error-certification is as high as $75 \%$. This result, indicating the improved robustness in the deterministic predictions from the mechanisms developed with the present strategy, appears systematically for all the obtained simplified mechanisms.

\section{Conclusions}

We proposed a general strategy for analysis and reduction of uncertain chemical kinetics models. We adopted an existing reduction method, based on computational singular perturbation (CSP) analysis, as a deterministic tool through which we propagated the uncertainty in the Arrhenius pre-exponential rate constants of the reference model to obtain the marginal probability of inclusion in the reduced model of each reaction. The simplified mechanisms are built by including those reactions whose marginal probability is higher than a given threshold. We defined probabilistic error measures to assess the performance of such simplified mechanisms taking uncertainty into account. We demonstrated the utilization of this strategy presenting an application where the uncertain kinetic model for the oxidation of n-butane was simplified based on different thresholds on marginal probabilities and several levels of accuracy determined by a number of tolerances on the reactions Importance Indices. We showed the probabilistic error figures for the obtained simplified mechanisms, highlighting the global trends of errors when varying probabilistic thresholds and tolerances on Importance Indices, and discussed some local non-monotonic behaviors of the errors that come from the complex way in which a given reaction mechanism/network performs when removing reactions/species. We have also illustrated the utility of the probabilistic reduction strategy for the general user who may be simply interested in robust deterministic predictions, showing that tight error tolerances enforced on simplified models obtained with deterministic approaches have a high probability of being missed because of the uncertainty in the detailed model. 
Ongoing work will further explore the various numerical and statistical aspects of the construction, focusing on its capabilities for examining the joint probability of sets of reaction. We will also explore additional detailed comparisons between deterministic and probabilistic analysis strategies, outlining further the utility of the present construction for identifying robust simplified models that are certifiably accurate over stated ranges of uncertainty.

\section{Acknowledgments}

This work was supported by the US Department of Energy (DOE), Office of Basic Energy Sciences (BES) Division of Chemical Sciences, Geosciences, and Biosciences. Sandia National Laboratories is a multiprogram laboratory operated by Sandia Corporation, a Lockheed Martin Company, for the United States Department of Energy under contract DE-AC04-94-AL85000. MV acknowledges the support of the Italian Ministry of University and Research (MIUR) and of CCRC/KAUST.

[1] M. Valorani, F. Creta, D. Goussis, J. Lee, H. Najm, Chemical Kinetics Simplification via CSP, Combustion and Flame 146 (2006) 29-51.

[2] M. Valorani, F. Creta, F. Donato, H. Najm, D. Goussis, Skeletal Mechanism Generation and Analysis for $n$-heptane with CSP, Proc. Comb. Inst. 31 (2007) 483-490.

[3] C. Homescu, L. R. Petzold, R. Serban, Error estimation for reduced-order models of dynamical systems, SIAM Review 49 (2) (2007) 277-299.

[4] B. Sonday, R. Berry, H. Najm, B. Debusschere, Eigenvalues of the Jacobian of a Galerkin-Projected Uncertain ODE System, SIAM J. Sci. Comp. 33 (2011) 1212-1233.

[5] M. Salloum, A. Alexanderian, O. Le Maître, H. Najm, O. Knio, Simplified CSP Analysis of a Stiff Stochastic ODE System, Computer Methods in Applied Mechanics and Engineering 217-220 (2012) 121-138. 
[6] C. Sun, J. Hahn, Model reduction in the presence of uncertainty model parameters, J. Process Control 16 (2006) 645-649, short communication.

[7] C. Sun, J. Hahn, Parameter reduction for stable dynamical systems based on Hankel singular values and sensitivity analysis, Chemical Engineering Science 61 (2006) 5393-5403.

[8] A. Antoulas, Approximation of Large-Scale Dynamical Systems, SIAM Publications, Philadelphia, PA, 2005.

[9] P. Benner, V. Mehrmann, D. Sorensen, Dimension Reduction of Large-Scale Systems, in: Lecture Notes in Computational Science and Engineering, Vol. 45, Springer-Verlag, Berlin/Heidelberg, Germany, 2005.

[10] S. Lam, D. Goussis, Conventional Asymptotics and Computational Singular Perturbation for Simplified Kinetics Modelling, in: M. Smooke (Ed.), Reduced Kinetic Mechanisms and Asymptotic Approximations for Methane-Air Flames, no. 384 in Springer Lecture Notes, Springer Verlag, 1991, Ch. 10, pp. 227-242.

[11] D. Goussis, S. Lam, A study of homogeneous methanol oxidation kinetic using csp, Proc. Comb. Inst. 24 (1992) 113-120.

[12] S. Lam, D. Goussis, Understanding complex chemical kinetics with computational singular perturbation, Proc. Comb. Inst. 22 (1988) 931-941.

[13] S. Lam, D. Goussis, The CSP Method for Simplifying Kinetics, International Journal of Chemical Kinetics 26 (1994) 461-486.

[14] P. D. Kourdis, R. Steuer, D. Goussis, Physical understanding of complex multiscale biochemical models via algorithmic simplification: Glycolysis in saccharomyces cerevisiae, Physica D: Nonlinear Phenomena 239 (2010) $1789-1817$.

[15] M. Valorani, H. Najm, D. Goussis, CSP Analysis of a Transient FlameVortex Interaction: Time Scales and Manifolds, Combustion and Flame 134 (1-2) (2003) 35-53. 
[16] H. Najm, M. Valorani, D. Goussis, J. Prager, Analysis of Methane-Air Edge Flame Structure, Combustion Theory and Modelling 14 (2) (2010) $257-294$.

[17] J. Prager, H. Najm, M. Valorani, D. Goussis, Structure of n-Heptane/Air Triple Flames in Partially-Premixed Mixing Layers, Combustion and Flame 158 (2011) 2128-2144.

[18] M. Valorani, F. Creta, A. Li Brizzi, H. Najm, D. Goussis, Surrogate Fuel Analysis and Reduction using Computational Singular Perturbation, AIAA-2008-1009AIAA $46^{\text {th }}$ AIAA Aerospace Sciences Meeting and Exhibit, Reno, NV.

[19] J. Prager, H. Najm, M. Valorani, D. Goussis, Skeletal Mechanism Generation with CSP and Validation for Premixed n-Heptane Flames, Proc. Comb. Inst. 32 (1) (2009) 509-517.

[20] M. Clyde, Bayesian model averaging and model search strategies (with discussion), in: J. Bernardo, J. Berger, A. Dawid, A. Smith (Eds.), Bayesian Statistics 6, Oxford University Press, 1999, pp. 157-185.

[21] J. A. Hoeting, D. Madigan, A. E. Raftery, C. T. Volinsky, Bayesian model averaging: a tutorial, Statistical Science 14 (4) (1999) 382-417.

[22] M. Valorani, F. Creta, F. Donato, H. Najm, D. Goussis, A csp-based skeletal mechanism generation procedure: Auto-ignition and premixed laminar flames in $n$-heptane/air mixtures, in: ECCOMAS CFD 2006, Delft, Holland, 2006.

[23] É. Hébrard, A. S. Tomlin, R. Bounaceur, F. Battin-Leclerc, Determining predictive uncertainties and global sensitivities for large parameter systems: A case study for n-butane oxidation, Proceedings of the Combustion Institute 35 (1) (2015) 607-616. 
[24] J. Warnatz, Resolution of gas phase and surface combustion chemistry into elementary reactions, in: Twenty-Fourth Symposium (International) on Combustion, Vol. 24, The Combustion Institute, 1992, pp. 553-579.

[25] B. Phenix, J. Dinaro, M. Tatang, J. Tester, J. Howard, G. McRae, Incorporation of Parametric Uncertainty into Complex Kinetic Mechanisms: Application to Hydrogen Oxidation in Supercritical Water, Comb. and Flame 112 (1998) 132-146.

[26] S. P. Sander, J. Abbatt, J. R. Barker, J. B. Burkholder, R. R. Friedl, D. M. Golden, R. E. Huie, C. E. Kolb, M. J. Kurylo, G. K. Moortgat, V. L. Orkin, P. H. Wine, Chemical kinetics and photochemical data for use in atmospheric studies, evaluation no. 17, Tech. Rep. JPL Publication 10-6, Jet Propulsion Laboratory, Pasadena, CA, http://jpldataeval.jpl.nasa.gov (2011).

[27] T. Turányi, T. Nagy, I. Zsély, M. Cserháti, T. Varga, B. Szabó, I. Sedyó, P. Kiss, A. Zempléni, H. Curran, Determination of rate parameters based on both direct and indirect measurements, Int. J. Chem. Kinetics 44 (2012) 284-302.

[28] D. Miller, M. Frenklach, Sensitivity Analysis and Parameter Estimation in Dynamic Modeling of Chemical Kinetics, Int. J. Chem. Kinetics 15 (1983) $677-696$.

[29] K. braman, T. Oliver, V. Raman, Bayesian analysis of syngas chemistry models, Combustion Theory and Modelling 17 (5) (2013) 858-887.

[30] M. Frenklach, H. Wang, M. J. Rabinowitz, Optimization and analysis of large chemical kinetic mechanisms using the solution mapping method combustion of methane, Progress in Energy and Combustion Science 18 (1) (1992) 47-73.

[31] M. Frenklach, A. Packard, P. Seiler, R. Feeley, Collaborative Data Process- 
ing in Developing Predictive Models of Complex Reaction Systems, Int. J. Chem. Kinetics 36 (2004) 57-66.

[32] M. Frenklach, Transforming data into knowledge - Process Informatics for combustion chemistry, Proc. Comb. Inst. 31 (1) (2007) 125-140.

[33] D. A. Sheen, H. Wang, Combustion kinetic modeling using multispecies time histories in shock-tube oxidation of heptane, Combustion and Flame 158 (4) (2011) 645-656.

[34] J. Prager, H. Najm, K. Sargsyan, C. Safta, W. Pitz, Uncertainty Quantification of Reaction Mechanisms Accounting for Correlations Introduced by Rate Rules and Fitted Arrhenius Parameters, Combustion and Flame 160 (2013) 1583-1593.

[35] A. S. Tomlin, E. Agbro, V. Nevrlỳ, J. Dlabka, M. Vašinek, Evaluation of combustion mechanisms using global uncertainty and sensitivity analyses: A case study for low-temperature dimethyl ether oxidation, International Journal of Chemical Kinetics 46 (11) (2014) 662-682.

[36] H. Wang, D. A. Sheen, Combustion kinetic model uncertainty quantification, propagation and minimization, Progress in Energy and Combustion Science 47 (2015) 1-31.

[37] M. Khalil, K. Chowdhary, C. Safta, K. Sargsyan, H. Najm, Inference of reaction rate parameters based on summary statistics from experiments, Proc. Comb. Inst.In press. doi:10.1016/j.proci.2016.08.058.

[38] S. D. Cohen, A. C. Hindmarsh, CVODE, a Stiff/Nonstiff ODE Solver in C, Comput. Phys. 10 (2) (1996) 138-143.

[39] C. Safta, H. Najm, O. Knio, TChem - a software toolkit for the analysis of complex kinetic models, Sandia Report SAND2011-3282, http://www.sandia.gov/tchem. 


\section{List of Figures}

1 Average Temperature (expressed in Kelvin), $\mathrm{C}_{4} \mathrm{H}_{10}, \mathrm{CO}$ and $\mathrm{OH}$ trajectories, with 2 standard deviation bounds, plotted against the normalized mixture specific entropy $\tilde{s} \ldots \ldots$. . . . . . 31

2 Temperature and normalized entropy $(\tilde{s})$ evolution in time for a number of samples, the box shows the normalized entropy evolution in time for $(1-\delta)<\tilde{s}<1$, with $\delta \approx 10^{-3} \ldots \ldots 32$

3 Self convergence of Max Error in $P_{\alpha}$ with increasing number of MC samples . . . . . . . . . . . . . . . . . . . . . 33

4 Number of retained/active species and reactions with increasing $\tau$ and $\theta \ldots \ldots \ldots \ldots \ldots \ldots \ldots$

5 Histograms of the probability of inclusion $\mathcal{P}_{r}^{\tau}$ for a representative choice of values for $\tau$. . . . . . . . . . . . . . . 35

6 Ignition delay times with their error bars at 2 standard deviations, for different $\tau$ and $\theta$ thresholds, compared with the detailed mechanism-based prediction in blue with error bounds at 2 standard deviations in red. $\theta$ thresholds are parametrizing each $\tau$-set, increasing from right to left. . . . . . . . . . . . . 36

$7 \quad 95 \%$ quantiles of the distributions of the relative errors in ignition delay time against the number of retained species. $\theta$ thresholds parametrize each $\tau$-set, increasing from right to left. . . . . . . 37

8 Average over the target species of the $\mathrm{L}_{2}$ un-weighted relative errors of the mean transient evolution against the number of retained species. $\theta$ thresholds parametrize each $\tau$-set, increasing from right to left. . . . . . . . . . . . . . . . . .

9 Average over the target species of the $\mathrm{L}_{2}$ un-weighted relative errors of the standard deviation of the transient evolution against the number of retained species. $\theta$ thresholds parametrize each $\tau$ set, increasing from right to left. . . . . . . . . . . . . .

10 PDF of $E_{3}(\lambda)$ for one of the probabilistically generated simplified mechanisms, and $\mathrm{PDF}$ of $E_{2}(\lambda)$ for a non-probabilistically generated simplified mechanism, with their error certification in the $\left\{\psi, X_{i}\right\}$ space $(\epsilon$, both in red). The highlighted areas are $P\left[E_{3}(\lambda)>\epsilon\right]$ and $P\left[E_{2}(\lambda)>\epsilon\right] \ldots \ldots \ldots$ 

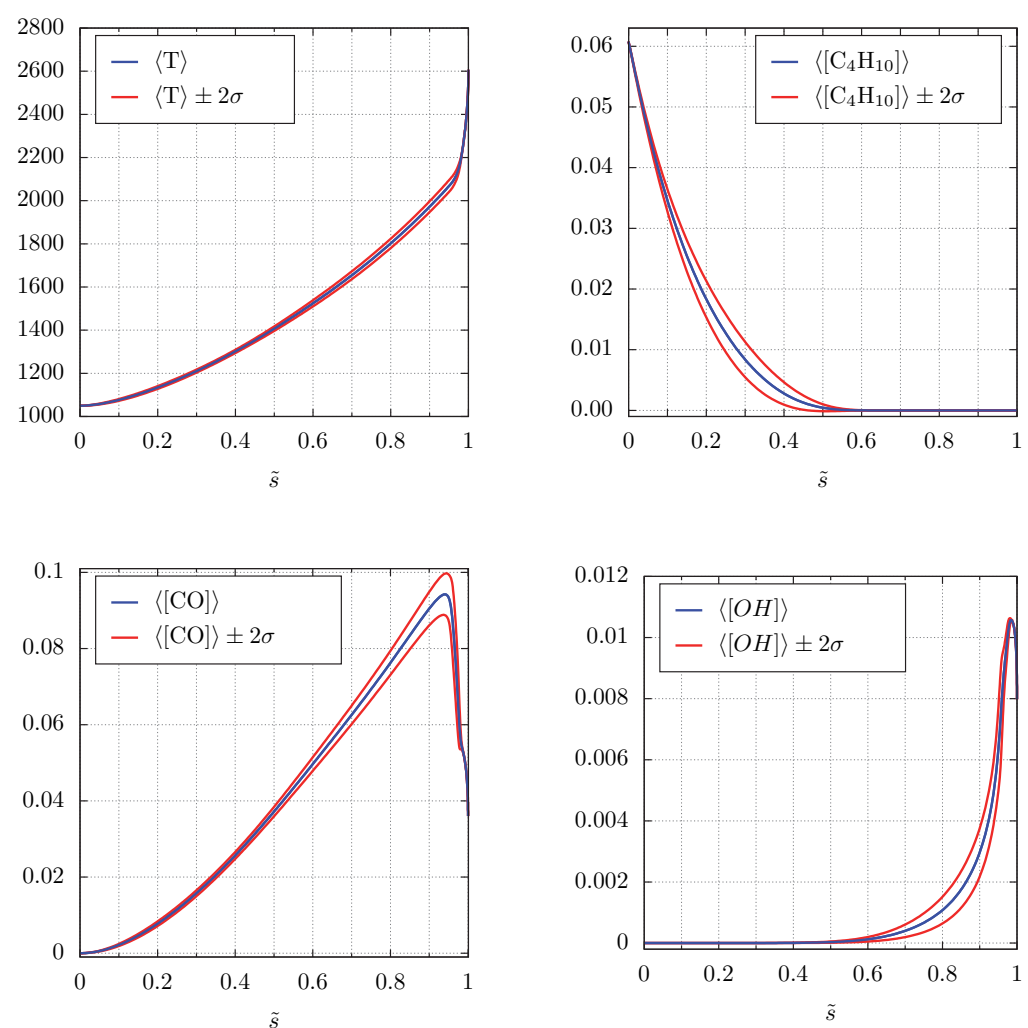

Figure 1: Average Temperature (expressed in Kelvin), $\mathrm{C}_{4} \mathrm{H}_{10}, \mathrm{CO}$ and $\mathrm{OH}$ trajectories, with 2 standard deviation bounds, plotted against the normalized mixture specific entropy $\tilde{s}$ 

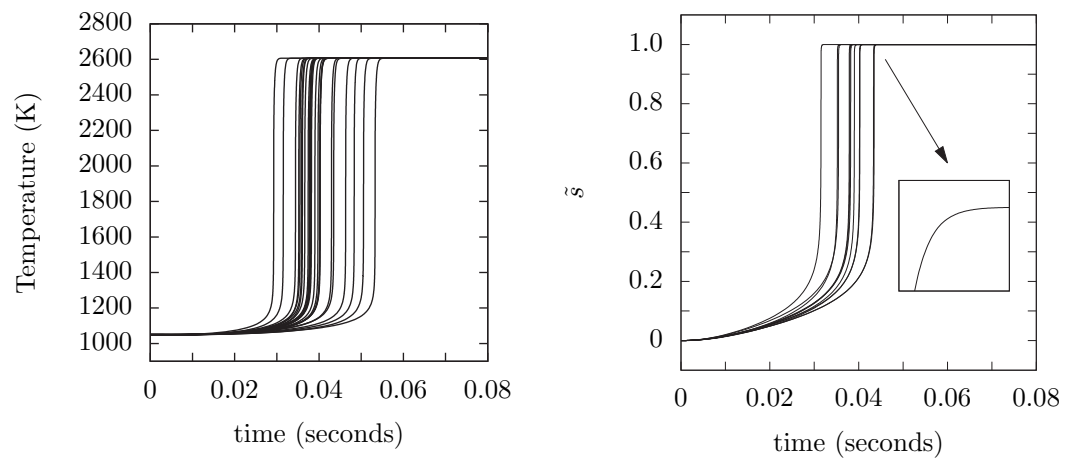

Figure 2: Temperature and normalized entropy $(\tilde{s})$ evolution in time for a number of samples, the box shows the normalized entropy evolution in time for $(1-\delta)<\tilde{s}<1$, with $\delta \approx 10^{-3}$ 


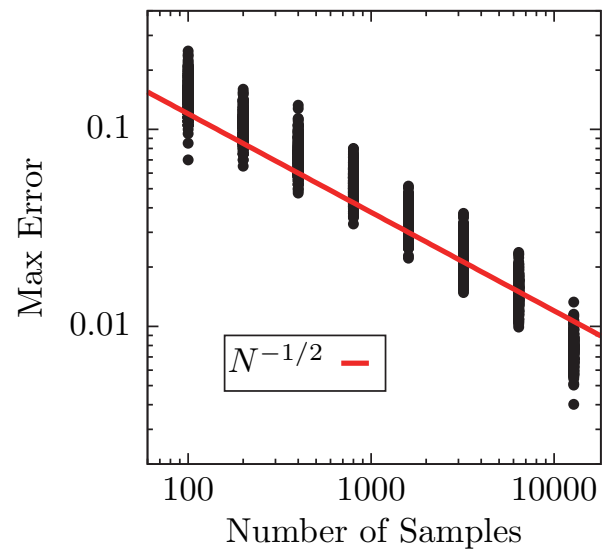

Figure 3: Self convergence of Max Error in $P_{\alpha}$ with increasing number of MC samples 

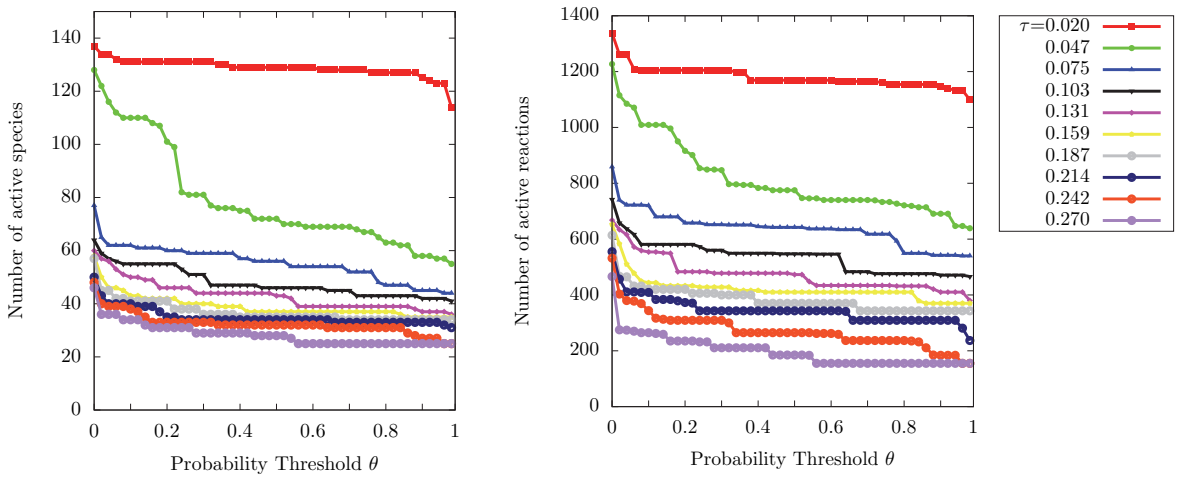

Figure 4: Number of retained/active species and reactions with increasing $\tau$ and $\theta$ 


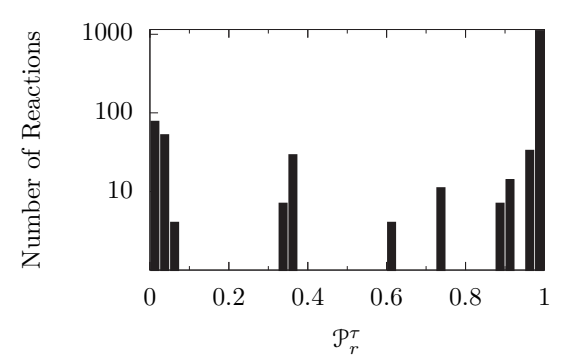

(a) $\tau=0.020$

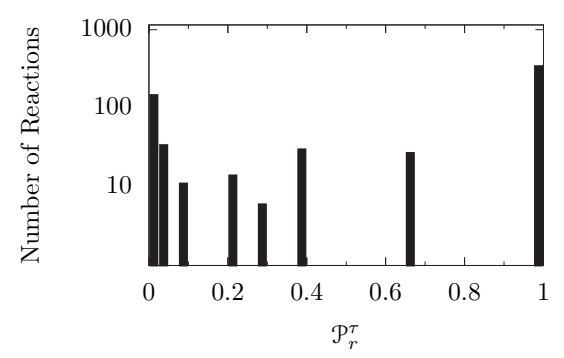

(b) $\tau=0.187$

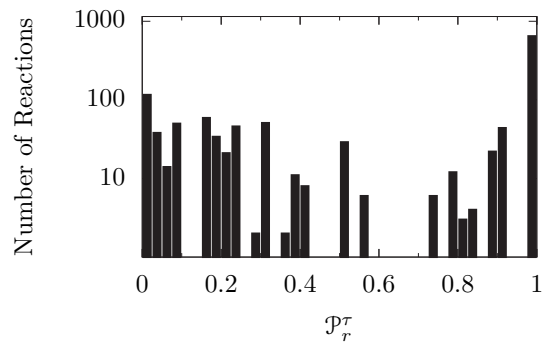

(c) $\tau=0.047$

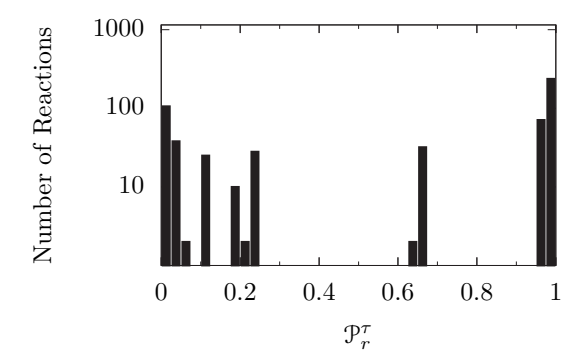

(d) $\tau=0.214$

Figure 5: Histograms of the probability of inclusion $\mathcal{P}_{r}^{\tau}$ for a representative choice of values for $\tau$. 


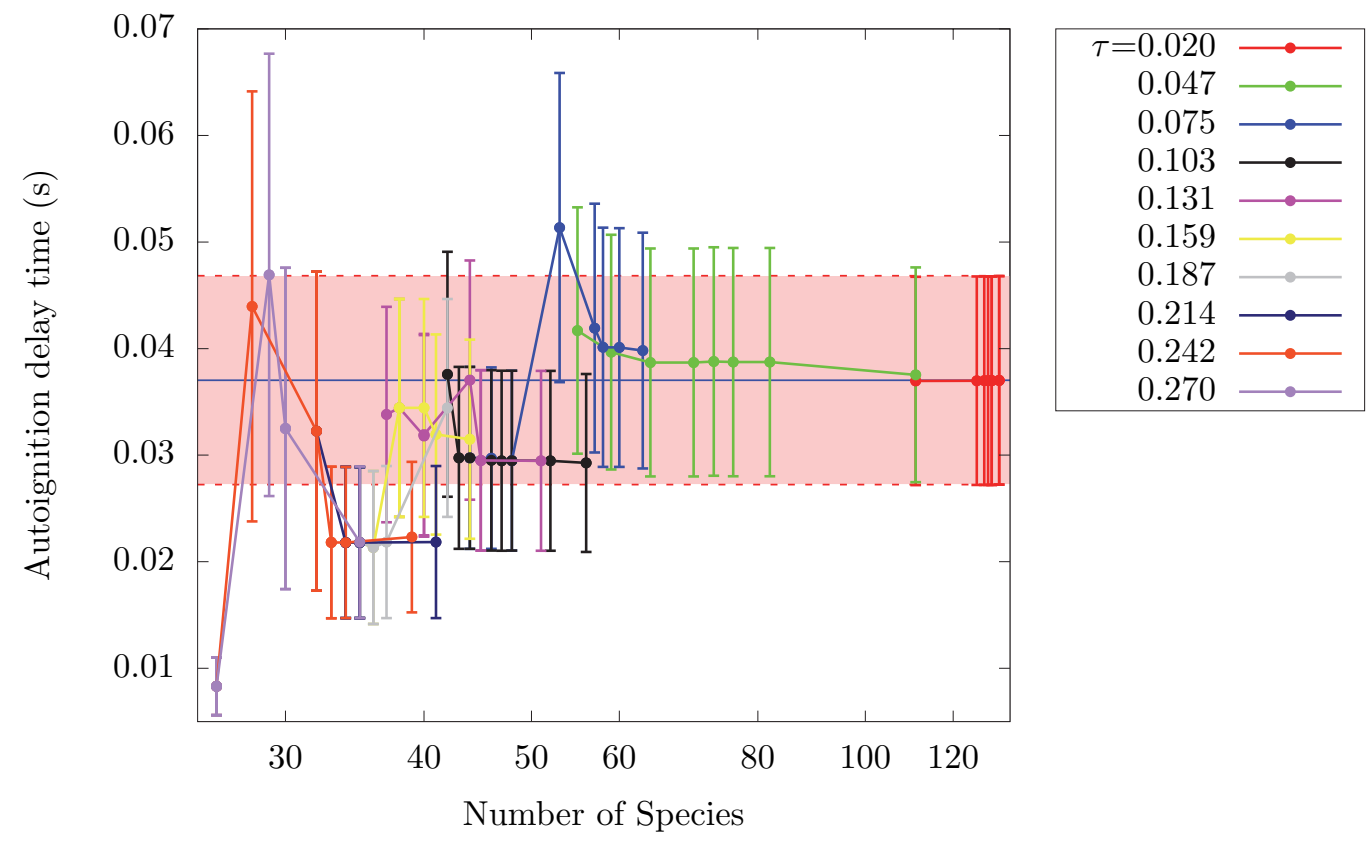

Figure 6: Ignition delay times with their error bars at 2 standard deviations, for different $\tau$ and $\theta$ thresholds, compared with the detailed mechanism-based prediction in blue with error bounds at 2 standard deviations in red. $\theta$ thresholds are parametrizing each $\tau$-set, increasing from right to left. 


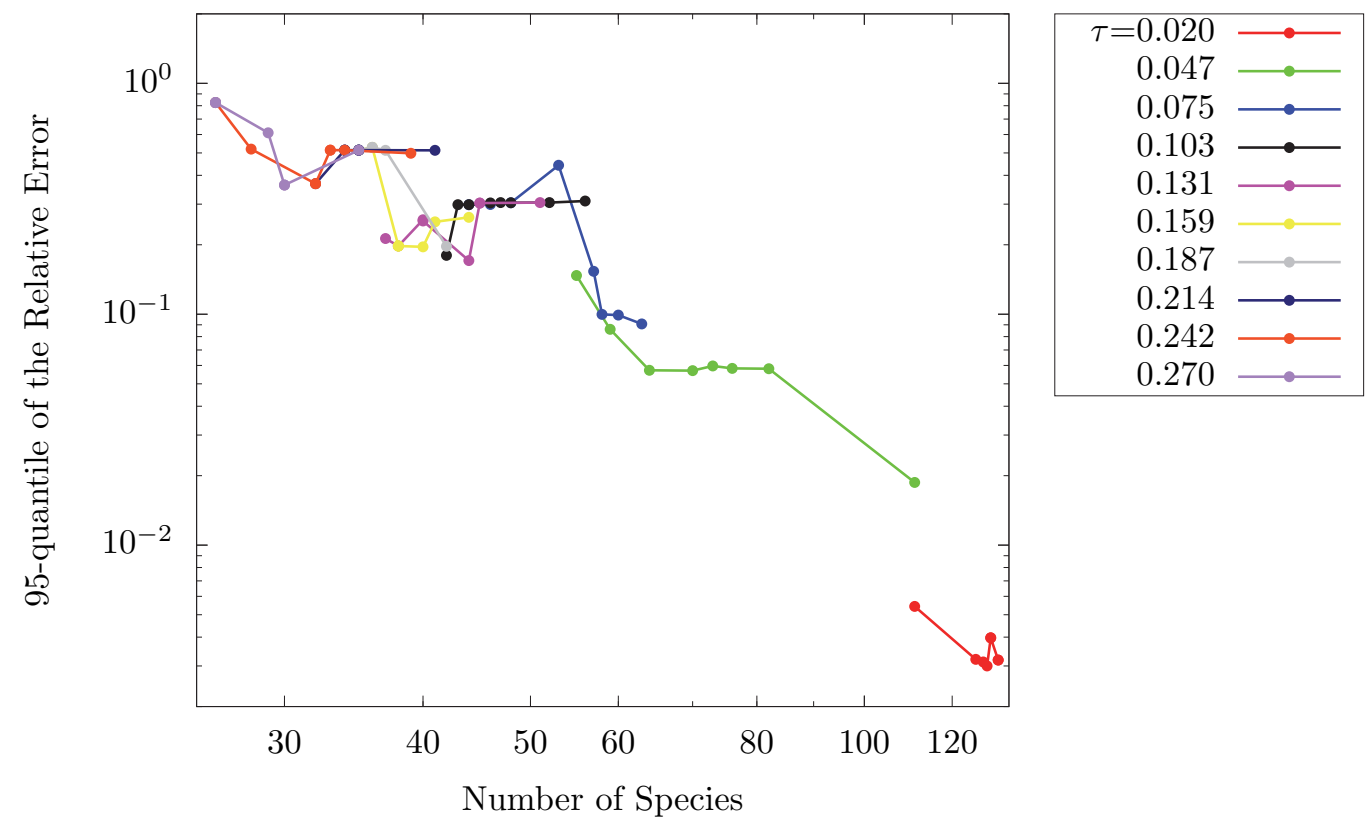

Figure 7: $95 \%$ quantiles of the distributions of the relative errors in ignition delay time against the number of retained species. $\theta$ thresholds parametrize each $\tau$-set, increasing from right to left. 


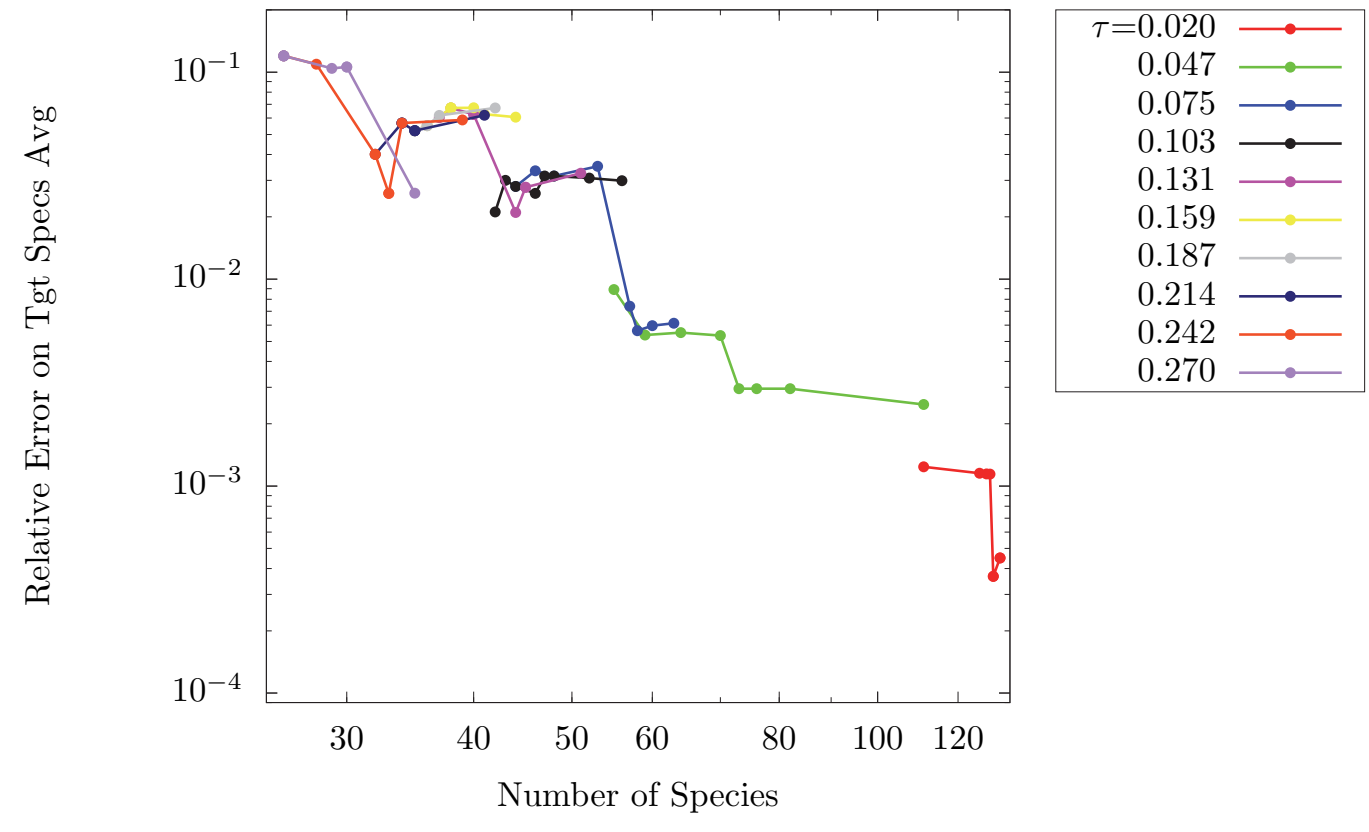

Figure 8: Average over the target species of the $\mathrm{L}_{2}$ un-weighted relative errors of the mean transient evolution against the number of retained species. $\theta$ thresholds parametrize each $\tau$-set, increasing from right to left. 


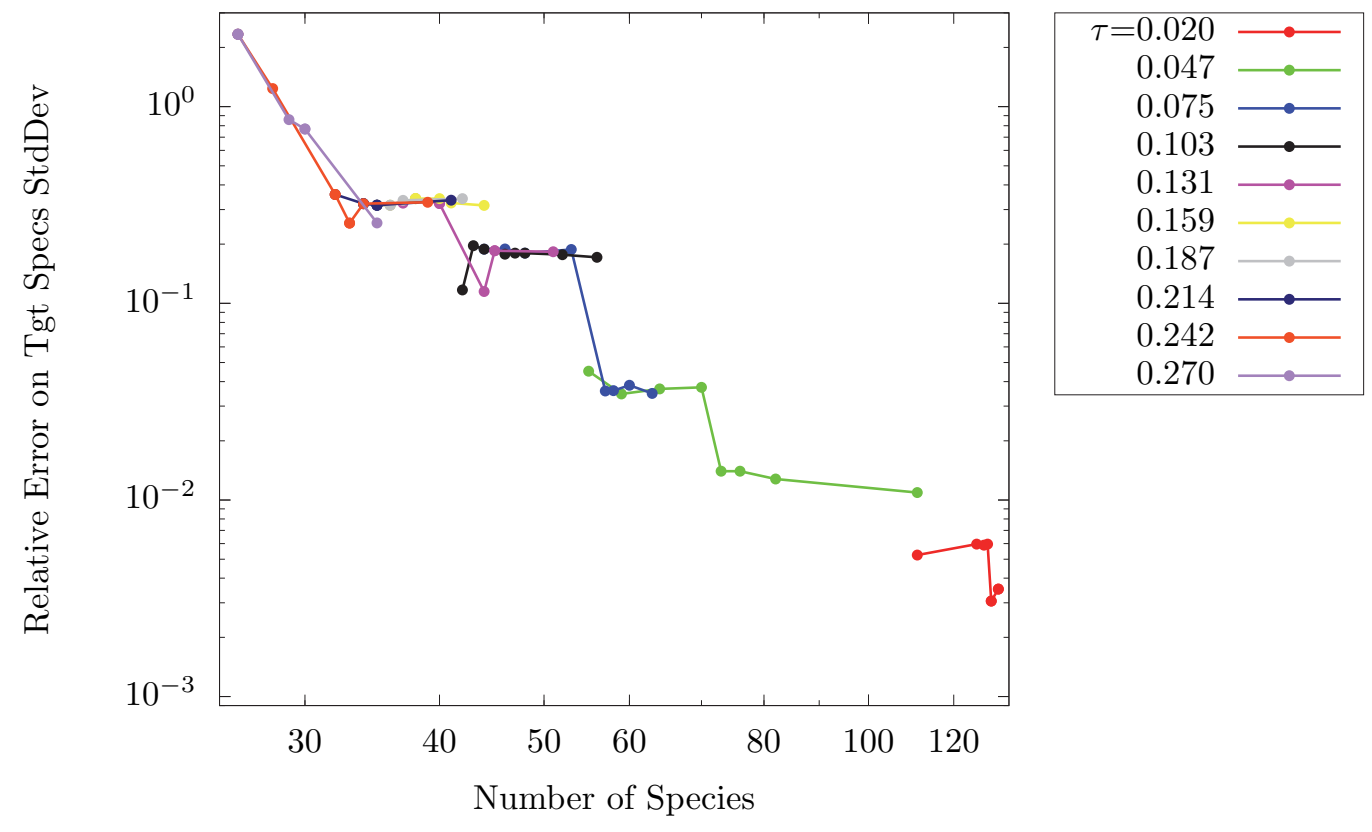

Figure 9: Average over the target species of the $\mathrm{L}_{2}$ un-weighted relative errors of the standard deviation of the transient evolution against the number of retained species. $\theta$ thresholds parametrize each $\tau$-set, increasing from right to left. 


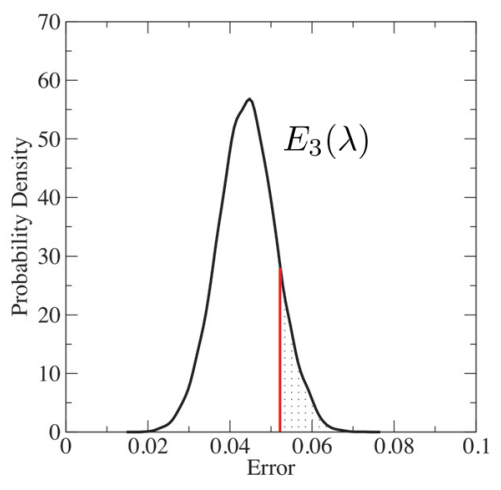

(a) $E_{3}(\lambda)$

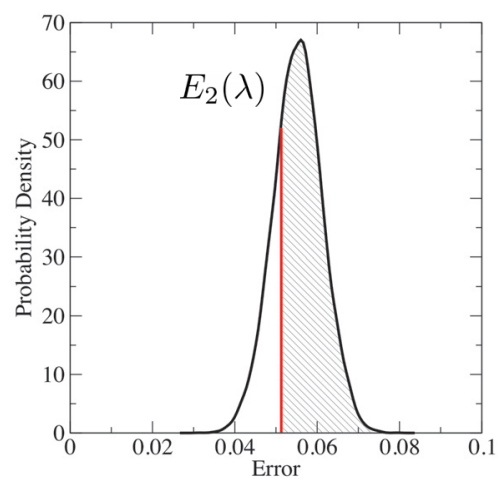

(b) $E_{2}(\lambda)$

Figure 10: $\mathrm{PDF}$ of $E_{3}(\lambda)$ for one of the probabilistically generated simplified mechanisms, and PDF of $E_{2}(\lambda)$ for a non-probabilistically generated simplified mechanism, with their error certification in the $\left\{\psi, X_{i}\right\}$ space $\left(\epsilon\right.$, both in red). The highlighted areas are $P\left[E_{3}(\lambda)>\epsilon\right]$ and $P\left[E_{2}(\lambda)>\epsilon\right]$ 